\title{
Dielectric Properties of Polymer Electrolyte Membranes Measured by Two-Port Transmission Line Technique
}

\author{
Z. Lu ${ }^{\text {a }}$, M. Lanagan ${ }^{\text {b }}$, E. Manias ${ }^{\text {a,c }}$ and D. D. Macdonald ${ }^{\text {a }}$ \\ ${ }^{a}$ Department of Materials Science and Engineering, Penn State University, University \\ Park, PA 16802 \\ ${ }^{\mathrm{b}}$ Department of Engineering Science and Mechanics, Penn State University, University \\ Park, PA 16802 \\ ${ }^{\mathrm{c}}$ Polymer Nanostructures Lab - Center for the Study of Polymer Systems, \\ Penn State University, University Park, PA 16802
}

\begin{abstract}
The microwave dielectric properties of perfluorosulfonic acid membranes are characterized by a two-port transmission line technique. Two dielectric relaxation modes are observed in the microwave region $(0.045-26 \mathrm{GHz})$ at $25^{\circ} \mathrm{C}$. The higher frequency process observed is identified as the cooperative relaxation of bulk-like water, whose amount was found to increase linearly with water content in the polymer. The lower-frequency process, characterized by longer relaxation times (20-70 picoseconds), is attributed to water molecules that are loosely bound to sulfonate groups. The loosely bound water amount was found to increase with hydration level at low water content, and levels-off at higher water contents. Flemion SH150, which has an equivalent weight of 909 g/equiv, displays higher dielectric strengths for both of these water modes compared to Nafion 117 (equivalent weight of 1100 g/equiv).
\end{abstract}

\section{Introduction}

\section{Dielectric Relaxation and AC Conduction in Perfluorosulfonic Membranes}

With the ever increasing interest in perfluorosulfonic acid (PSA) membranes, such as Nafion and Flemion, there is a growing demand for dielectric characterization of these materials towards a quantitative understanding of the dynamics of water molecules and protons within the membranes. PSA membranes are essentially aqueous electrolytes because they are only operational when sufficient amount of water is absorbed around the sulfonic acid groups. Dielectric relaxation spectroscopy (DRS), which monitors the cooperative motion of molecular assemblies, provides a powerful tool for characterization of these materials.

The dielectric properties of a material is described by the complex relative permittivity spectrum,

$$
\varepsilon^{*}(\omega)=\varepsilon^{\prime}(\omega)-j \varepsilon^{\prime \prime}(\omega)
$$


with $\varepsilon^{\prime}(\omega)$ denoting the relative permittivity (or dielectric constant), $\varepsilon^{\prime \prime}(\omega)$ for the dielectric loss (or absorption), and $j=\sqrt{-1}$. The dielectric properties of polymer electrolytes can yield important information on the molecular structure of the system and the physical state of water in these materials (1-4). Furthermore, the dielectric relaxation behavior provides insight on the impact of the polymer environments on the cooperative solvent dynamics, which have important ramifications on water and proton transport in these membranes (5-7).

Despite their obvious importance, the available dielectric data for PSA polymer electrolytes are rather limited. Mauritz and coworkers $(8,9)$ reported constant temperature dielectric relaxation analyses of Nafion membranes imbibed with various ions. Tsonos et al. $(10,11)$ studied the dielectric relaxation of $\mathrm{K}^{+}$exchanged Nafion at $25{ }^{\circ} \mathrm{C}$. Howevre, these investigations were limited to low frequencies $(\mathrm{f}<10 \mathrm{MHz}$ ) and no detailed information about discrete water relaxations was obtained. Conversely, the frequencies for dielectric relaxations in aqueous electrolytes at ambient temperature are normally observed in the microwave region, which requires special instrumentation. Additional difficulty associated the dielectric measurements of aqueous electrolytes originates from the fact that the only experimentally accessible variable is the total dielectric loss, which contains the contribution of the electrical conductivity $(\sigma)$, expressed as

$$
\eta^{\prime \prime}(\omega)=\varepsilon^{\prime \prime}(\omega)+\eta_{\sigma}^{\prime \prime}=\varepsilon^{\prime \prime}(\omega)+\frac{\sigma}{\omega \varepsilon_{0}}
$$

where $\varepsilon_{0}$ is the permittivity of the vacuum. Obviously, the Ohmic loss, $\eta_{\sigma}^{\prime \prime}$, dominates the total dielectric loss at lower frequencies, limiting the spectral range accessible to experiments (4). Therefore, there is an increasing demand for accurate high frequency (microwave) dielectric characterization techniques capable of robust measurement of the dielectric properties of various electrolytes including aqueous and polymeric materials.

The selection of a suitable microwave dielectric characterization technique is dependent upon the delicate properties, frequency range, sample geometry and available equipment [12]. Transmission and resident methods are the two general measurement categories and each has their advantage. The resonant technique typically measures dielectric properties at a single frequency and is useful for low dielectric loss specimens. Transmission techniques (13-16), on the other hand, have swept frequency capability over a wide frequency range (10 $\mathrm{MHz}$ to $26 \mathrm{GHz}$ in this study). In addition, a wide range of permittivity values can be measured (but dielectric loss measurements are limited to above 0.005). A two-port transmission line technique is selected for the study of high dielectric loss PSA membranes.

In our previous work, we measured the dielectric spectra of hydrated Nafion 117 with the two-port transmission line method and identified at least two water relaxations in the frequency range of $0.1-26 \mathrm{GHz}$ (4). In order to study the dielectric relaxation behavior of the membranes having different equivalent weight (EW), we measure microwave dielectric spectra of another perfluorated sulfonic acid membrane, Flemion SH150, which has a similar chemical structure to Nafion, but has a lower equivalent weight (EW= 909 g/equiv versus $\mathrm{EW}=1100$ for Nafion 117). It is expected that $\mathrm{EW}$ is an important factor in 
determining the phase separated structure of the hydrated membrane and thus the dynamics of protons and water molecules within the membrane.

\section{Theoretical Background for Transmission Line Method}

Two-port transmission techniques are widely used for high loss specimens because of their high accuracy $(17,18)$. In general, these techniques make use of the reflected and/or transmitted waves by and through a dielectric-filled transmission line in order to analytically or numerically determine the dielectric properties of the material (Figure 1). In this setup, a dielectric sample is installed in a coaxial air-filled line with characteristic impedance $\mathrm{Z}_{0}$. Within the sample region the line will assume a new characteristic impedance $Z=Z_{0} \sqrt{\mu^{*} / \varepsilon^{*}}$, where $\varepsilon^{*}$ and $\mu^{*}$ are respectively the complex relative permittivity and permeability of the sample. The reflection coefficient $(\Gamma)$ at the interface between the air-filled line and dielectric-filled line when the material sample is infinite in length is given by

$$
\Gamma=\frac{Z-Z_{0}}{Z+Z_{0}}=\frac{\sqrt{\mu^{*} / \varepsilon^{*}}-1}{\sqrt{\mu^{*} / \varepsilon^{*}}+1}
$$

The air-filled waveguide sections (regions I and III in Figure 1) are included here, in order to take into account the offset between the calibration plane and the measurement plane.

The fundamental propagation mode for waves in a coaxial system is the transverse electromagnetic wave (TEM). The spatial distribution of the electric field in the regions I, II, and III, for an incident field normalized to 1, can be written as (16):

$$
\begin{aligned}
& E_{I}=\exp \left(-\gamma_{0} z\right)+C_{1} \exp \left(\gamma_{0} z\right) \\
& E_{I I}=C_{2} \exp (-\gamma z)+C_{3} \exp (\gamma z) \\
& E_{I I I}=C_{4} \exp \left(-\gamma_{0} z\right)
\end{aligned}
$$

where $\gamma$ is the complex propagation constant given by

$$
\gamma=\alpha+j \beta=j \omega \sqrt{\varepsilon^{*} \varepsilon_{0} \mu^{*} \mu_{0}}
$$

where $\varepsilon_{0}$ and $\mu_{0}$ are the permittivity and magnetic permeability of vacuum, and $\omega$ is the angular frequency ( $\omega=2 \pi f) \cdot \varepsilon^{*} \varepsilon_{0}$ and $\mu^{*} \mu_{0}$ are the absolute permittivity and magnetic permeability of the medium. In the air-filled regions I and III, $\gamma$ reduces to

$\gamma_{0}=j \omega \sqrt{\varepsilon_{0} \mu_{0}}$. The constants $\mathrm{C}_{\mathrm{i}}$ are determined by appropriate matching of the tangential electric and magnetic fields at all boundaries. 
The reflection $\left(\mathrm{S}_{11}\right)$ and transmission scattering parameters $\left(\mathrm{S}_{21}\right)$ are obtained by solving Eqs. [4]-[6]. The explicit expressions are given by [31]

$$
\begin{aligned}
& S_{11}=R_{1}^{2}\left[\frac{\Gamma(1-\exp (-2 \gamma L)}{1-\Gamma^{2} \exp (-2 \gamma)}\right] \\
& S_{21}=R_{1}^{2}\left[\frac{\exp (-\gamma L)\left(1-\Gamma^{2}\right)}{1-\Gamma^{2} \exp (-2 \gamma)}\right]
\end{aligned}
$$

where $R_{1}$ and $R_{2}$ are the reference plane transformations, and are expressed by

$$
\begin{aligned}
& R_{1}=\exp \left(-\gamma_{0} L_{1}\right) \\
& R_{2}=\exp \left(-\gamma_{0} L_{2}\right)
\end{aligned}
$$

For high dielectric loss materials (such as Nafion or Flemion membranes) measurement of $S_{21}$ allows for a higher accuracy than that of $S_{11}$ (18). Therefore, in this work, the dielectric properties are determined by measuring the frequency dependence of the transmission $\mathrm{S}$-parameter $\mathrm{S}_{21}$. It should be noted that the analytical solution for the permittivity is difficult to obtain from Eq. 9, and numerical methods that are exclusive to $\mathrm{S}_{21}$ are used instead [17].

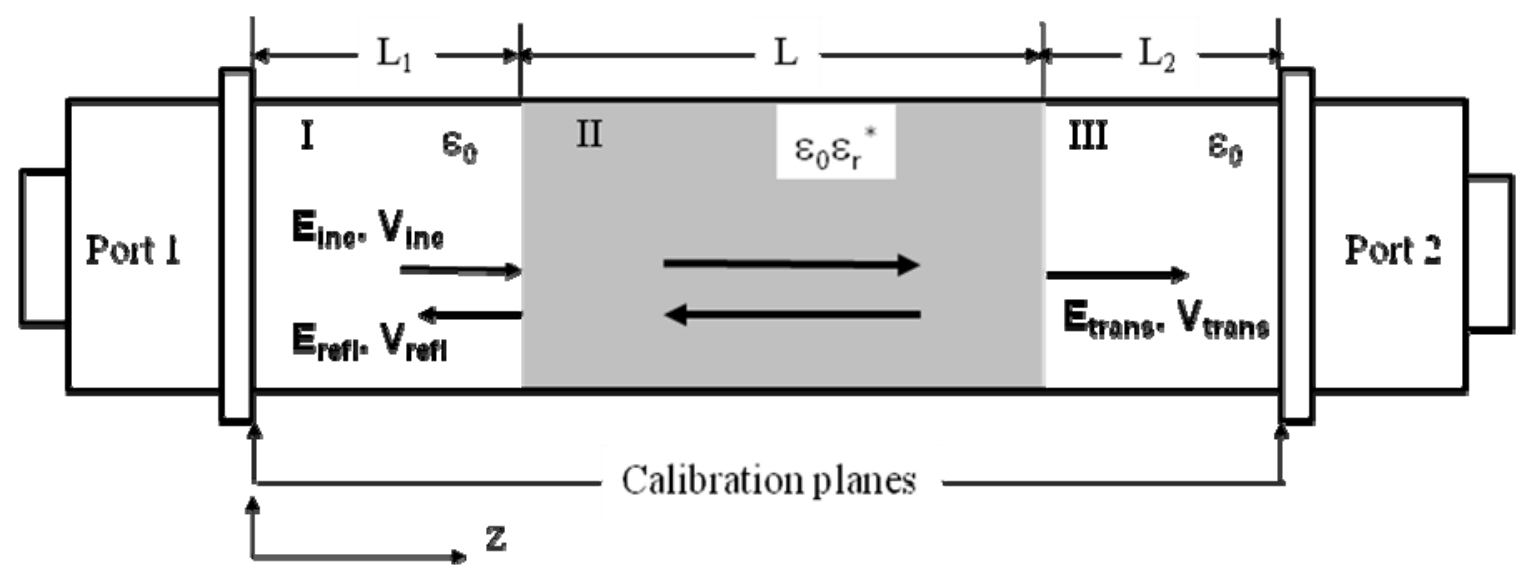

Figure 1. The incidence (inc), reflected (refl) and transmitted (trans) electric field/voltage distributions in the measurement setup of the two-port transmission line technique. Port 1 and port 2 denote calibration reference plane positions.

In this numerical procedure, $\varepsilon^{\prime}(\omega)$ and $\varepsilon^{\prime \prime}(\omega)$ are separately determined from the magnitude $\left(\left|S_{21}\right|\right)$ and phase $(\phi)$ of $S_{21}\left(S_{21}=\left|S_{21}\right| e^{j \phi}\right)$. The real part of the permittivity $\left(\varepsilon^{\prime}(\omega)\right)$ is first calculated from the phase through the phase factor $\beta$ as

$$
\phi=-\beta L=-\frac{2 \pi \sqrt{\varepsilon^{\prime}(\omega)}}{\lambda_{0}} L
$$


where $\lambda_{0}$ is the wavelength in vacuum. After determination of $\varepsilon^{\prime}(\omega)$, the imaginary part of the complex permittivity $\varepsilon^{\prime \prime}(\omega)$ is iteratively obtained from the magnitude of $S_{21}$. The data processing is carried out with a self-built computer program. This particular technique assumes that internal reflections caused by the dielectric/air interface are negligible, which is valid for high loss samples with a sufficient length to attenuate internally reflected microwave.

\section{Dielectric Measurements of Flemion SH150}

A vector network analyzer HP8510C, with a broad band sweep generator synthesized signals from $45 \mathrm{MHz}$ to $26 \mathrm{GHz}$, was used to measure the complex transmission parameter $\mathrm{S}_{21}$. A $7 \mathrm{~mm}$ coaxial line (10 cm long) was connected to HP8510C through two standard transmission lines with impedance of $50 \Omega$. Before measurement, the system

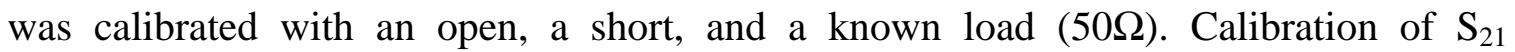
magnitude was made by performing a simple through connection with air as the dielectric medium. Each S-parameter measurement is subsequently corrected for the reference plane transformation (Eqs. 10 and 11). The dielectric properties of the specimen are then calculated from these S-parameters.

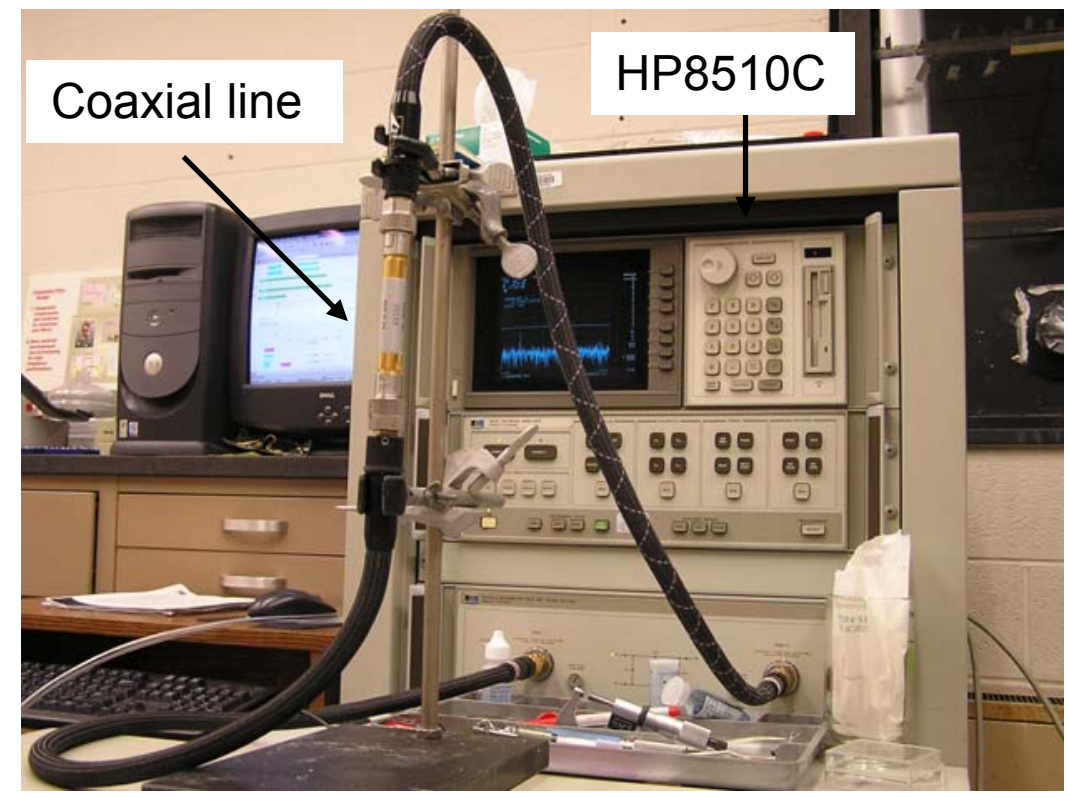

Figure 2. S-parameter measurement setup using HP8510C vector network analyzer

The Flemion membranes were treated by boiling in $3 \% \mathrm{H}_{2} \mathrm{O}_{2}$, rinsing in boiling water, then boiling in $0.5 \mathrm{M} \mathrm{H} 2 \mathrm{SO} 4$, and finally rinsing in boiling water. The membranes were then hydrated at $25^{\circ} \mathrm{C}$ under three RHs, $33 \%, 75 \%$ and $100 \%$, to contain a water content ( $\lambda$, denoting the numbers of absorbed water molecules per $-\mathrm{SO}_{3} \mathrm{H}$ site) of 4.5, 7.1 and 13.5, respectively. The pretreated Flemion membranes were carefully wrapped around 
the inner conductor of the coaxial line. After the installation of the sample, the coaxial line (containing the sample) was conditioned again in the same salt solution to restore the membrane water content. The coaxial line was then quickly connected to the network analyzer HP8510C and the transmission S-parameter $\left(\mathrm{S}_{21}\right)$ was collected over the entire frequency range. The measurement usually lasted less than 5 minutes and no variation in membrane water content was found.

Further, in order to establish a reference for the various relaxing components, we also did microwave DRS measurements on polytertrafluoroethylene (PTFE) film (McMaster, $0.003^{\prime \prime}$ thick, electronic grade) and dry Flemion membrane. The "dry" membrane was obtained by drying under vacuum at $120^{\circ} \mathrm{C}$ for 12 hours. The water content for the dry membrane is estimated as $\lambda \sim 1$.

\section{Results and discussion}

\section{$\underline{\text { Microwave Dielectric Spectra of Flemion SH150 }}$}

Figure 3 shows the dielectric spectra of the dry Flemion membrane along with the results of the "dry" Nafion 117 (4) and PTFE. No relaxation process can be identified from the absorption spectra in the studied frequency range. Dry Flemion SH150 and dry Nafion 117 have very similar values of permittivity and dielectric loss, which indicates that the chemical structure and the equivalent weight have little effect on the dielectric properties of dry PSA membranes. The higher values of $\varepsilon^{\prime}$ and $\varepsilon^{\prime \prime}$ of both dry PSA compared to those of PTFE are due to the presence of sulfonic acid groups in these membranes.

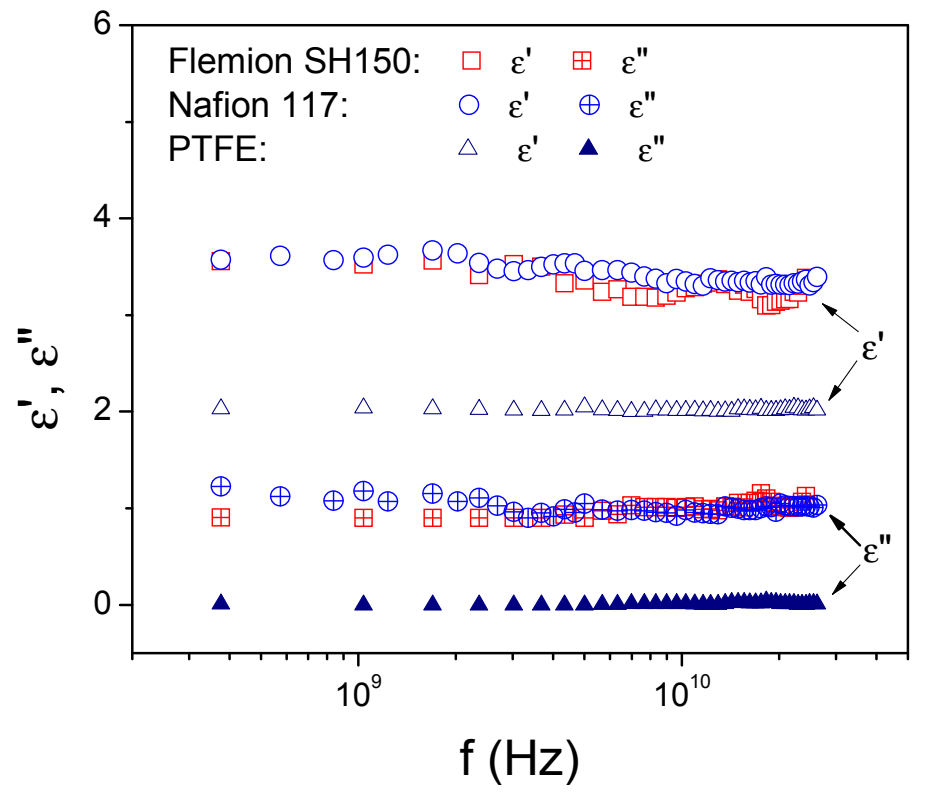

Figure 3. Relative permittivity, $\varepsilon^{\prime}(\omega)$, and dielectric loss spectrum, $\varepsilon^{\prime \prime}(\omega)$, of dry Flemion $\mathrm{SH} 150$ at $25^{\circ} \mathrm{C}$. The dielectric spectra for dry Nafion 117 are also included. 
The dielectric spectra of hydrated Flemion membranes are remarkably different from those of the dry samples. Figure 4 shows the relative permittivity $\varepsilon^{\prime}(\omega)$ and the total loss $\eta^{\prime \prime}(\omega)$ of Flemion samples with water contents of $\lambda=4.5,7.1$ and 13.5, equilibrated at RH of $33 \%, 75 \%$ and $100 \%$, respectively. The contribution of the conductivity due to the long-range motion of $\mathrm{H}^{+} / \mathrm{H}_{2} \mathrm{O}$ is clearly shown in the low frequency range and follows an inverse frequency $\left(f^{-1}\right)$ dependence.
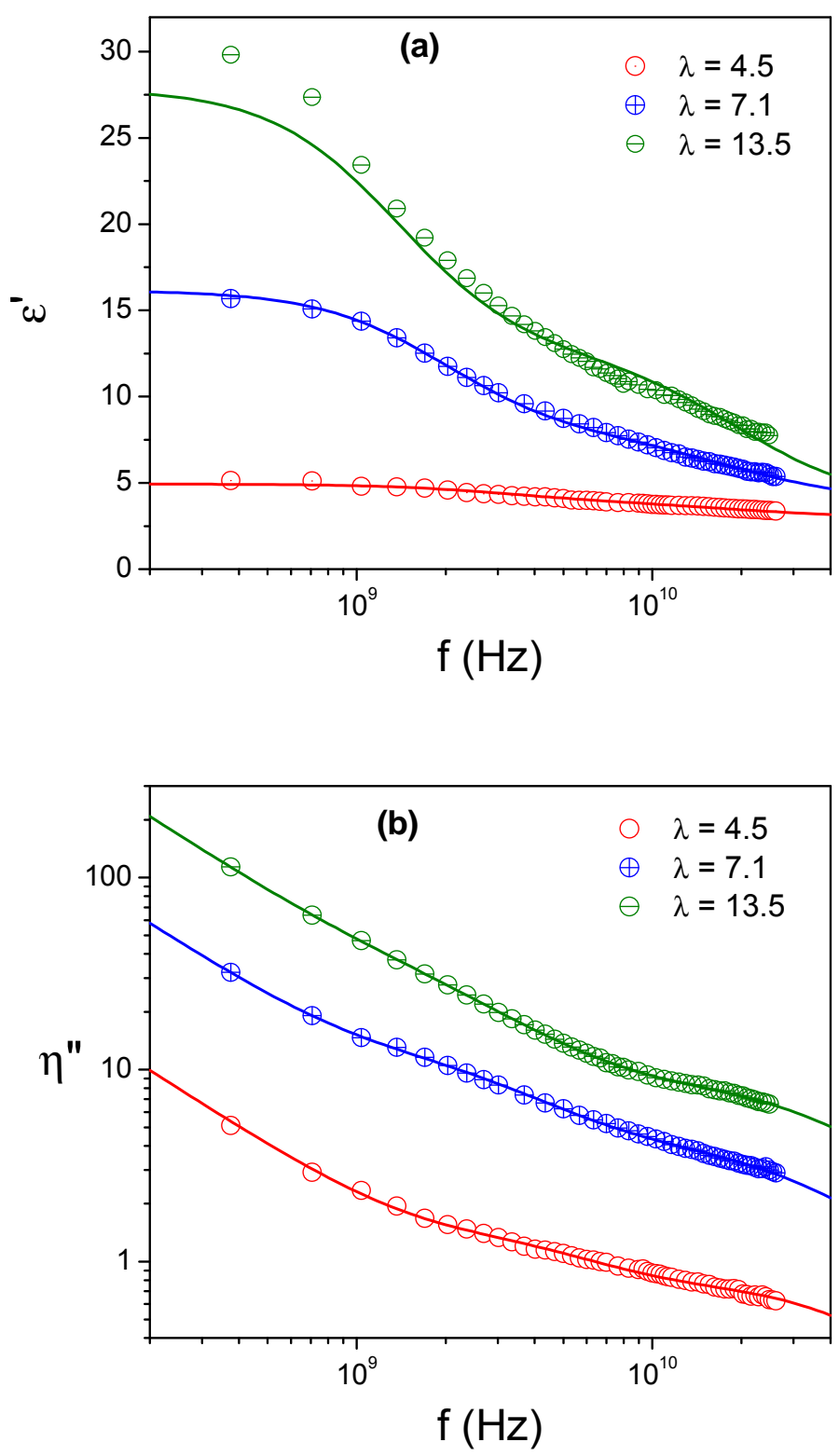

Figure 4. Permittivity spectra vs. frequency at $25 \mathrm{oC}$ for Flemion SH150 with water content of $\lambda=4.5,7.1$ and 13.5: (a) relative permittivity $\varepsilon^{\prime}(\omega)$ and (b) total loss $\eta^{\prime \prime}(\omega)$. The solid lines represent the corresponding best fits by two Debye processes. 


\section{Data Analysis}

For each measurement, the total loss of the sample, $\eta^{\prime \prime}(\omega)$, together with the dielectric permittivity, $\varepsilon^{\prime}(\omega)$, was numerically calculated from the measured $S_{21}$ spectrum. According to Eq. 2, the true dielectric loss of the electrolytes, $\varepsilon^{\prime \prime}(\omega)$, can only be obtained from $\eta^{\prime \prime}(\omega)$ after the correction for the conductivity contribution. The (dc) conductivity, $\sigma$, is treated as an adjustable parameter in the data analysis.

After subtraction of the conductivity contribution, the reduced dielectric spectra $\varepsilon^{*}(\omega)$ are fitted to a superposition of multiple Havriliak-Negami equations or variants thereof $(19,20)$.

$$
\mathcal{E}^{*}(\omega)=\varepsilon_{\infty}+\sum_{i=1}^{n} \frac{\Delta \varepsilon_{i}}{\left[1+\left(j \omega \tau_{i}\right)^{1-\alpha_{i}}\right]^{\beta_{i}}} \text { with } \Delta \varepsilon_{i}=\varepsilon_{s i}-\varepsilon_{\infty i}, 0 \leq \alpha_{i} \leq 1,0 \leq \beta_{i} \leq 1
$$

where $\mathrm{n}$ is the number of separable dispersion steps, $\varepsilon_{s i}$ and $\varepsilon_{\infty i}$ are the low and highfrequency permittivity limits for the $\mathrm{i}^{\text {th }}$ process from the lower frequency side, respectively, and $\omega=2 \pi f$ is the angular frequency, $\tau$ is the dielectric relaxation time, and $\alpha$ and $\beta$ are shape parameters for the distribution of relaxation times. This general equation can be reduced to three well-known models as limiting cases: the Debye equation ( $\alpha=0, \beta=1$ ), the Cole-Cole equation ( $0 \leq \alpha<1, \beta=1$ ), and the Davidson-Cole equation ( $\alpha=0,0<\beta \leq 1$ ). The complex permittivity spectra, $\varepsilon^{*}(\omega)$, are analyzed by simultaneously fitting $\varepsilon^{\prime}(\omega)$ and $\varepsilon^{\prime \prime}(\omega)$ with a least-square procedure, based on the Levenberg-Marquardt algorithm (for more details see also Reference 4).

For Flemion samples with $\lambda=4.5$ and 7.1, the complex permittivity spectra were best fit with a sum of two Debye relaxation processes $(\alpha=0, \beta=1)$. The spectra for $\lambda=13.5$ did not yield satisfactory fitting of the lower-frequency peak despite that the higherfrequency peak is fitted with good certainty. The same situation has also been found for Nafion 117 equilibrated at 100\% RH (4). The noise of the permittivity data at the lower frequencies in the PSA samples with the highest water content may be due to the water condensation inside the coaxial line during sample preparation.

\section{Dielectric Relaxations of Water in PSA Membranes}

The true dielectric loss $\varepsilon^{\prime \prime}(\omega)$ is obtained when the contribution of the conductivity is subtracted from the total loss (Eq. 2). Figure 5 shows the dielectric response of the hydrated Flemion membranes at $25{ }^{\circ} \mathrm{C}$ with three water contents, along with the fitted curves corresponding to two Debye relaxations. These two relaxation processes are essentially identical to the two relaxation modes observed in hydrated Nafion 117 (4). The higher-frequency process is attributed to the dielectric relaxation of the bulk-like water in the membrane. This dielectric relaxation corresponds to the ordinary water behavior dominated by water-water hydrogen bonding $(21,22)$. The lower-frequency process in Figure 5, having a longer relaxation time ranging from 20 to70 ps, is attributed to water molecules loosely bound to the sulfonic groups. Due to the broad distribution of 
environments within the PSA membrane, the loosely bound water can exist in many different local environments: (i) water in second hydration shell surrounding the strongly bound water to the sulfonates, (ii) water in hydrophilic channels connecting the hydrated ionic clusters, or (iii) water at the interface between the polymer and the bulk-like liquid water as well as water confined in the hydrophobic domains of the membrane (i.e., hydrophobic hydration water). A third type of water, which had been observed with the broadband DRS to display a relaxation in the $\mathrm{MHz}$ range, cannot be captured in the microwave rage (4). The type of water molecules are tightly bound to the sulfonic sites (also called hydration water).

Figure 6 shows the comparison of the dielectric dispersion strength $(\Delta \varepsilon)$ between Flemion and Nafion 117. The bars for the loosely bound water at $\lambda=13.5$ for Flemion and $\lambda=12$ for Nafion samples are due to data scattering at low frequencies and indicate ranges of $\Delta \varepsilon$ that can yield equally good fits. From this figure, the loosely bound water increases with increased hydration and levels-off at higher $\lambda$, while the bulk-like water is low at low water content and then increases almost linearly with $\lambda$. It can be reasonably assumed that the bulk-like water will become dominant at the higher hydration levels. It is also clear from Figure 6 that Flemion shows higher dielectric strengths than Nafion for both the loosely bound water and the bulk-like water. This is in agreement with the expectation that at lower EW larger size and/or larger number of hydrated ionic clusters can be formed $(6,23)$. However, since Flemion SH150 and Nafion 117 are synthesized by different manufacturers, no further analysis is possible on the quantifying the effect of equivalent weight.

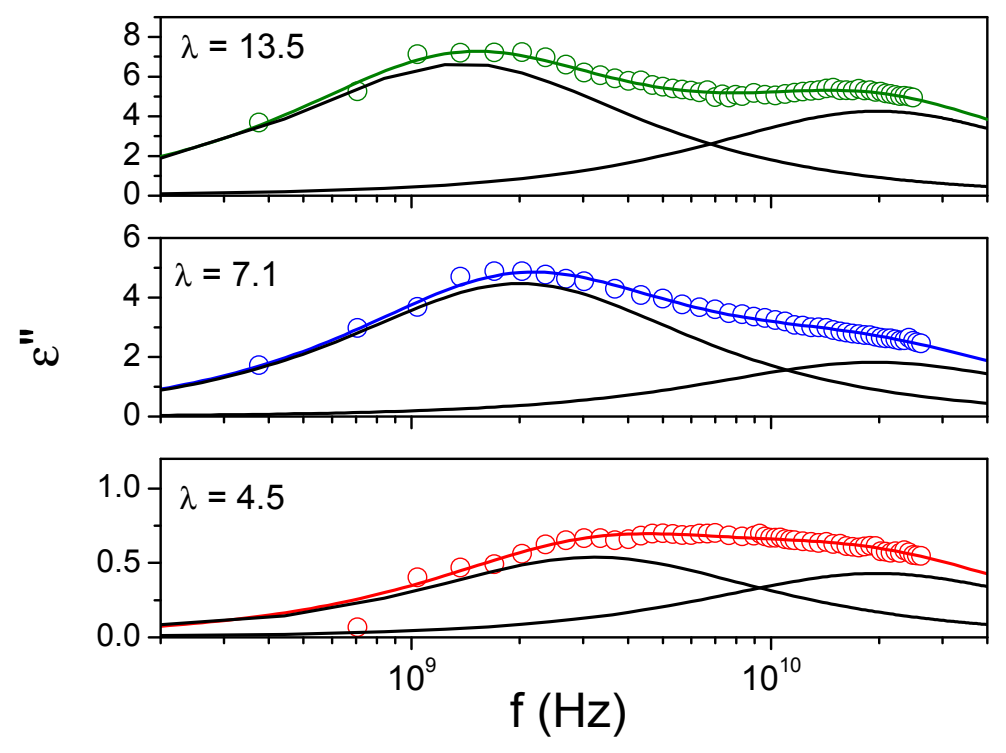

Figure 5. Dielectric loss $\varepsilon^{\prime \prime}(\omega)$ vs. frequency at $25{ }^{\circ} \mathrm{C}$ for Flemion SH150 with water content of $\lambda=4.5,7.1$ and 13.5. The solid lines represent the corresponding best fits by two Debye processes. The faster process (high-frequency peak) corresponds to bulklike water and the slower process (lower-frequency peak) to loosely bound water. 


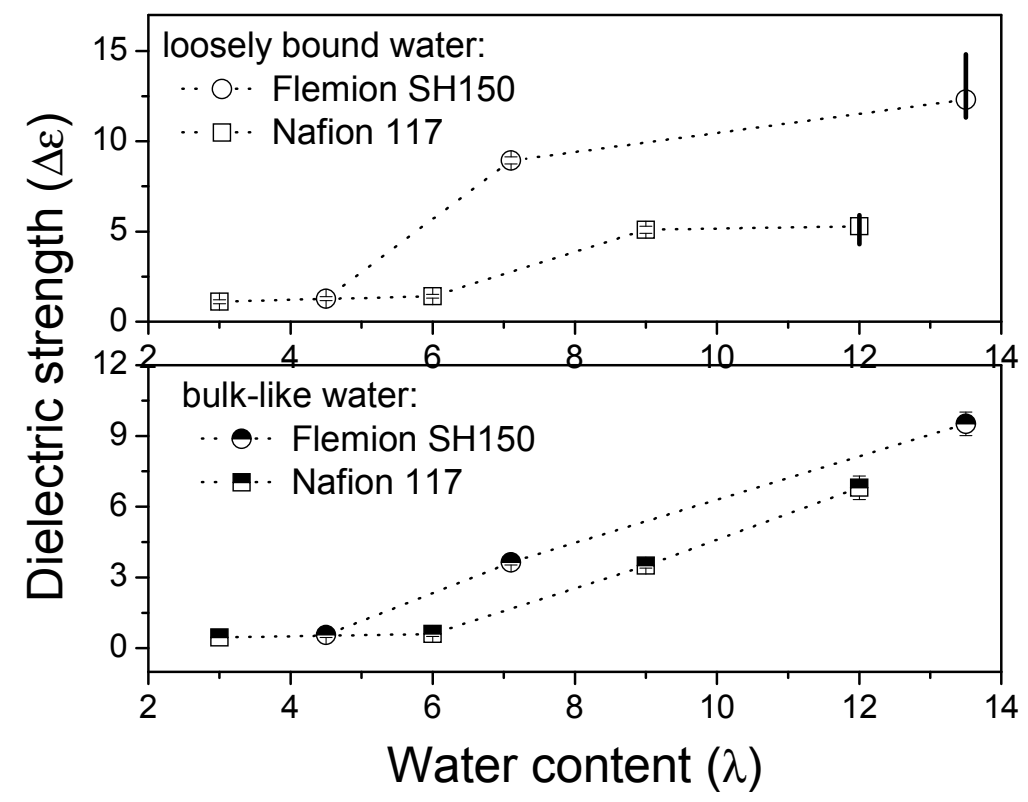

Figure 6. The comparison of the dielectric strengths between Flemion SH150 and Nafion 117 samples at $25{ }^{\circ} \mathrm{C}$. The bars for the loosely bound water at $\lambda=13.5$ for Flemion and $\lambda$ $=12$ for Nafion samples are due to data scattering at low frequencies.

\section{Conclusion}

In this work, the dielectric relaxation spectra of dry and hydrated Flemion SH150 membrane, which has a similar chemical structure to Nafion 117, but has a lower equivalent weight (EW= 909 g/equiv vs. EW=1100 g/equiv), are measured with a twoport transmission line technique over the frequency range of $45 \mathrm{MHz}$ to $26 \mathrm{GHz}$ at $25{ }^{\circ} \mathrm{C}$. The permittivity spectra are well-fitted by a superposition of two Debye relaxation processes. The higher-frequency process shows the characteristic dynamics of liquid water and is attributed to the cooperative relaxation of free/bulk-like water. The lowerfrequency peak, characterized by longer relaxation times of 20-70 picoseconds, is attributed to water molecules that are loosely bound to the membrane. The amount of the free/bulk-like water, quantified through the dielectric strength of each corresponding relaxation mode, increases linearly with increasing water content, while the loosely bound water increases with increased hydration and levels-off at higher water contents. Flemion SH150 displays higher dielectric strengths for the two water modes and therefore higher dielectric constant than Nafion 117, which may indicate the effect of the equivalent weight on the polymer structure, particularly on the ionic cluster regions.

\section{Acknowledgments}

Z.L. acknowledges Mr. Steve Perini for his help in the experimentation and Dr. Khalid Rajab for his assistance in software. The authors gratefully acknowledge financial support for this work by International Fuel Cells Inc. through subcontract no. 3540OB via Contract No. DE-FCo4-02AL67608 and by the U.S. Department of Energy via Contract 
No. DE-FG02-07ER46371 at Penn State University. E.M. additionally acknowledges support from the National Science Foundation (NSF grant \# DMR-0602877).

\section{References}

1. R. Paul and S. J. Paddison, J. Phys. Chem. B, 108, 13231 (2004).

2. R. Paul and S. J. Paddison, J. Chem. Phys., 123, 224704 (2005).

3. S. J. Paddison, R. Paul, T. A. Zawodzinski, Jr, J. Electrochem. Soc., 147, 617 (2000).

4. Z. Lu, G. Polizos, D. D. Macdonald, E. Manias, J. Electrochem. Soc., 155, B163 (2008).

5. S. J. Paddison, Annu. Rev. Mater. Res., 33, 289 (2003).

6. M. Saito, N. Arimura, K. Hayamizu, T. Okada, J. Phys. Chem. B, 108, 16064 (2004).

7. S. J. Paddison and R. Paul, Phys. Chem. Chem. Phys., 4, 1158 (2002).

8. K. A. Mauritz, Macromolecules, 22, 4483 (1989).

9. Z. D. Deng and K. A. Mauritz, Macromolecules, 25, 2739 (1992).

10. C. Tsonos, L. Apekis, P. Pissis, J. Mater. Sci., 35, 5957 (2000).

11. C. Tsonos, L. Apekis, P. Pissis, J. Mater. Sci., 33, 2221 (1998).

12. L. Chen, V. K. Varadan, Microwave Electronics: Measurement and Materials Characterization, John Wiley and Sons (2004).

13. J. Baker-Jarvis, M. D. Janezic, R. G. Geyer, IEEE Trans. Instrum. Meas., 43, 711 (1994).

14. S. J. Paddison, D. W. Reagor, T. A. Zawodzinski, Jr., J. Electroanal. Chem., 459, 91 (1998).

15. S. Jenkins, T. E. Hodgetts, R. N. Clarke, A. W. Preece, Meas. Sci. Technol., 1, 691 (1990).

16. J. Baker-Jarvis, E. J. Vanzura, W. A. Kissick, IEEE Trans. Microwave Theory Techn., 38, 1096 (1990).

17. M. T. Lanagan, J. H. Kim, D. C. Dube, S. J. Jang, R. E. Newnham, Ferroelectrics, 82, 91 (1988).

18. M. T. Lanagan, Ph.D. Dissertation, the Pennsylvania State University (1987).

19. S. Havriliak and S. Negami, Polymer, 8, 161 (1967).

20. F. Kremer and A. Schönhals, Broadband Dielectric Spectroscopy, Springer, New York (2002).

21. U. Kaatze, J. Chem. Eng. Data, 34, 371 (1989).

22. J. Barthel, K. Bachhuber, R. Buchner, H. Hetzenauer, Chem. Phys. Lett., 165, 369 (1990).

23. G. Gebel, Polymer, 41, 5829 (2000). 